\title{
Le médecin qui prenait ses distances
}

\section{Daniel Lüthi}

Journaliste indépendant et photographe, formateur en communication, Berne

Il devient président en pleine pandémie: qui l'aurait imaginé au moment de son élection en novembre dernier?! Et maintenant, peu avant son entrée en fonction, il se livre à un entretien d'un genre particulier, alors que le coronavirus terrorise le monde entier. Mais à une condition, avait précisé Henri Bounameaux: que nous soyons tous les deux asymptomatiques. Une des premières choses qu'il a dites: "Gardons nos distances, c'est probablement la seule chose qui soit utile aujourd'hui, avec le lavage régulier des mains.»

\section{Personnellement touché}

Un médecin qui entonne "gardons nos distances» comme si c'était une maxime: qui aurait pris cela comme une évidence il y a quelques semaines?! Ma rencontre avec Henri Bounameaux illustre bien les différents aspects de ce précepte. Tout d'abord, mon interlocuteur est personnellement concerné: un membre de sa famille est atteint du coronavirus. Et lui-même se demande quand il pourra revoir ses petits-enfants.

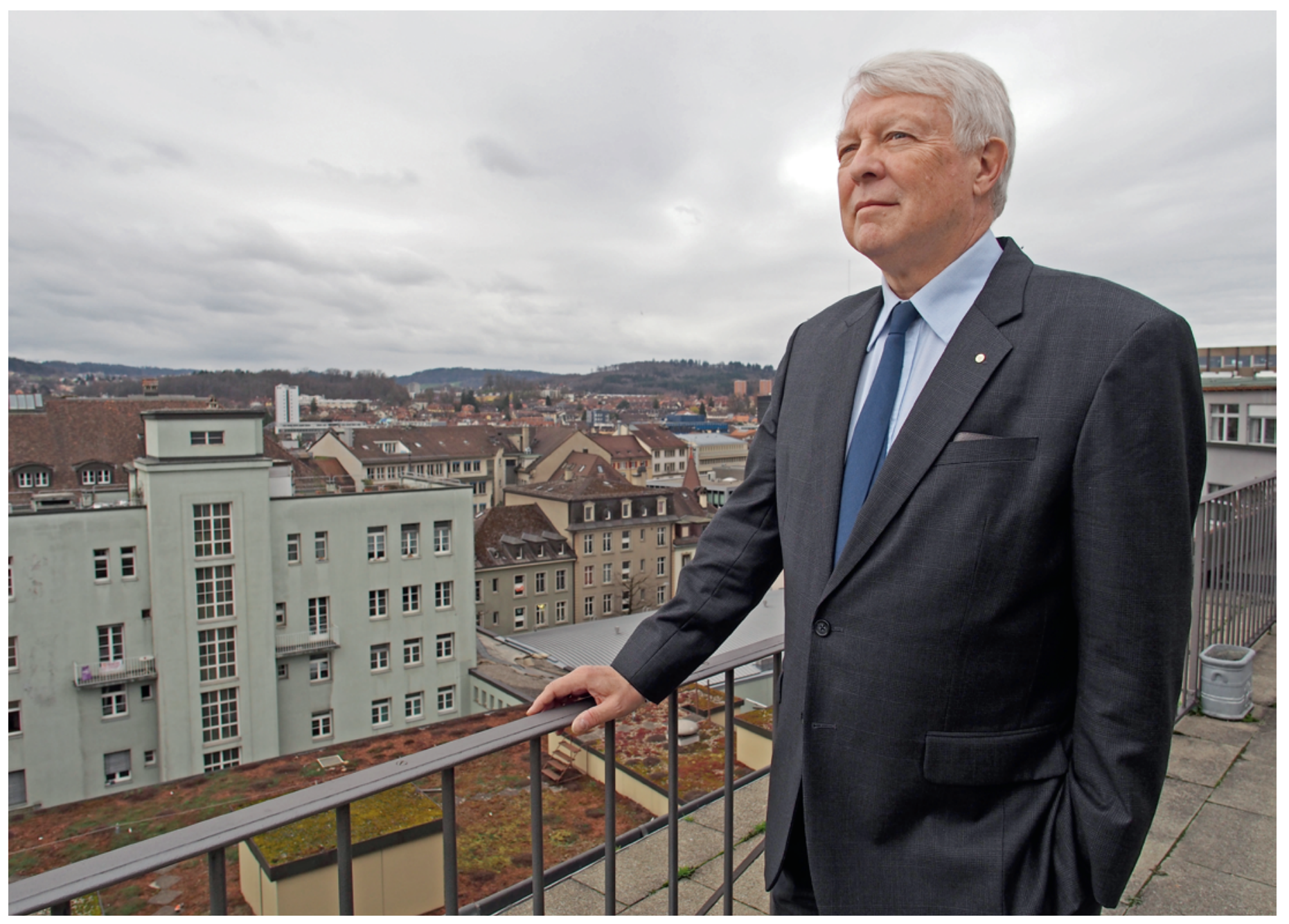


En tant médecin, il en saisit évidemment l'enjeu: «Nous devons vivre avec le risque de tomber malade. Et plus on vieillit, plus on se rapproche du terme.» Certes, mais Henri Bounameaux sait parfaitement ce qui se passe en coulisses: "Je n'ai pas envie de me retrouver aux soins intensifs avec une pneumonie virale.»

Nos emplacements respectifs sont clairement désignés, nous nous asseyons à une distance suffisante l'un de l'autre. Devoir se tenir éloignés alors que le besoin de rapprochement entre les gens est plus fort que jamais actuellement. Nous avons sciemment renoncé à nous serrer la main, bien que ce soit une convention sociale très ancrée dans nos mœurs. Ce qui nous fait entrer dans le vif du sujet, qui intéresse beaucoup Henri Bounameaux: «Nombreux sont ceux qui craignent le virus - ce sont souvent les mêmes qui négligent la vaccination contre la grippe saisonnière qui cause chaque année 8000 à 10000 décès en Suisse.» A cet instant, l'homme d'habitude plutôt réservée et distinguée se met presque hors de lui: «La vaccination est un des plus grands progrès de la médecine. S'y opposer est une ineptie!» Une attitude «scientifico-sceptique» de plus en plus fréquente qu'il entend bien combattre

\section{«La vaccination est un des plus grands progrès de la médecine.»}

dans ses nouvelles fonctions: «Même certains médecins et soignants adoptent un comportement individualiste et oublient le risque de contamination qu'ils font courir aux autres.» Le nouveau président de l'ASSM prend clairement ses distances vis-à-vis des antivaccins.

\section{Se distancer d'une interdiction}

Autre priorité de son mandat qui le fait bondir: «Ceux qui s'opposent par principe à l'expérimentation animale s'opposent en fait à la recherche tout court et donc au progrès médical», avance Henri Bounameaux. «'aime bien les animaux, mais on ne devrait pas expérimenter directement sur l'humain.» Evidemment, chaque expérience doit être justifiée et réalisée dans le respect de règles éthiques claires, mais: «L'interdiction de l'expérimentation animale serait dangereuse.»

Henri Bounameaux passe d'ailleurs une bonne partie de son temps libre avec les animaux. "Je suis cavalier», raconte-t-il. Quatre fois par semaine, il se promène dans la campagne sur son demi-sang français. Sa fille et une de ses petites-filles font du concours complet, une discipline combinant dressage, saut et cross. Les chevaux ont toujours joué un grand rôle dans la famille, souligne le médecin.

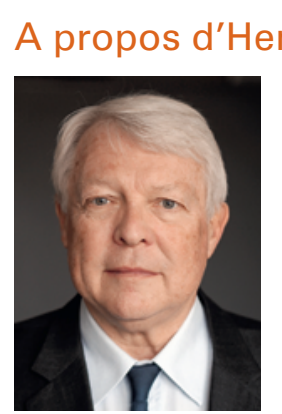

Bounameaux

Le professeur Henri Bounameaux est né en 1953 à Liège, Belgique. II a effectué le début de sa scolarité à Lubumbashi, dans le sud de l'actuelle République démocratique du Congo, où son père était médecin et professeur de médecine. A l'âge de 14 ans, sa famille s'établit à Bâle où il suivra ses études de médecine. Diplômé en 1978, il se spécialise en médecine interne et en angiologie. De 1993 à 2015, il dirige le nouveau service d'angiologie et d'hémostase des Hôpitaux universitaires de Genève (HUG). II est promu professeur ordinaire en 2002 et devient directeur du Département de médecine interne. De 2011 à 2019, il est doyen de la faculté de médecine de Genève et exerce aux HUG la fonction de directeur de l'enseignement et de la recherche. En novembre 2019, il est élu nouveau président de l'Académie Suisse des Sciences Médicales (ASSM), avec entrée en fonction le $1^{\text {er }}$ mai 2020.

Henri Bounameaux est marié, père de deux filles et six fois grand-père. II vit à Satigny, dans la campagne genevoise.

La famille? Sa femme et ses deux filles. Elles ont dû souffrir de tous ses engagements professionnels, concède le retraité encore bien actif, et s'il a un regret, c'est sans doute celui-là.

Aujourd'hui, il peut voir les choses avec davantage de recul sans devoir s'occuper de chaque détail. Là aussi, «je peux maintenant prendre de la distance».

\section{Se distancer des privilèges}

Mais il doit aussi s'engager dans ses nouvelles fonctions. "Beaucoup de mes collègues ouvrent un cabinet privé à leur retraite», dit Henri Bounameaux, songeur, «je n'aurais plus le 'feu sacré', pour soigner des patients, je l'ai fait pendant 40 ans». Maintenant, il veut bâtir des ponts, entre la science et la société, par exemple. Et entre la profession médicale et les autres acteurs de la santé: "Nous devons abolir les privilèges.» Les médecins, hommes et femmes, sont de toute façon débordés, il faudra bien qu'ils délèguent certaines tâches à d'autres. "Il n'est pas nécessaire d'avoir fait dix ou quinze ans d'études pour examiner un pied diabétique», affirme le médecin retraité. S'il veut ainsi introduire en Suisse le système cubain, comme cela lui a déjà été demandé, glisse-t-il d'un ton narquois: "Cela prendra plus de temps.»

Mais il compte combattre avec tact un certain mercantilisme de la profession et les actes médicaux inutiles. "A Genève, nous avons 40 installations d'IRM, avons-nous besoin d'une $41^{\text {e }}$ », dit-il pour illustrer son 
propos. Nous devrions nous inspirer des modèles hollandais ou scandinaves, selon Henri Bounameaux. «Les spécialistes ont leur place à l'hôpital, les généralistes au sein de la population. Cela fonctionne et

\section{"L'interdiction de l'expérimentation animale serait dangereuse.»}

coûte moins cher.» Pour lui, le problème n'est pas le revenu individuel, mais la répartition de l'enveloppe, une répartition que «nous devons revoir sur le long terme».

La relève scientifique en Suisse est aussi un thème important: il faut inciter les jeunes médecins à se consacrer à la recherche, et cette activité doit être décemment rémunérée. «L'hôpital doit prendre en charge une moitié pour le travail clinique, l'université l'autre moitié pour les activités de recherche.» L'ASSM doit investir dans cette problématique: «L'Académie doit soutenir essais et expériences et aider à trouver à cette fin les financements appropriés.»

\section{Se distancer du quotidien}

En tant qu'instance supérieure, l'Académie est une référence. C'est pourquoi le président de l'ASSM doit se distancer de l'actualité du quotidien et prendre de la hauteur pour avoir une vue d'ensemble. Henri Bounameaux en est persuadé. Nous voici donc revenus à la question principale de cet entretien, celle de la pandémie en cours et de ses conséquences possibles. Les questions médico-éthiques de fond constituent le fer de lance de l'ASSM. Qu'en est-il du renforcement de la stigmatisation par la crise du coronavirus de certains groupes au sein de la société déjà stigmatisés? Cette situation exceptionnelle va-t-elle accroître l'égoïsme des individus et la segmentation d'une société déjà très individualiste? Et ainsi la solitude de toujours davantage d'êtres humains? «Bonnes questions», répond Henri Bounameaux. "J'ai déjà réfléchi à organiser une retraite du comité de l'ASSM sur le thème des épidémies et de leurs aspects éthiques.» Mais il aime aussi le concret. Par exemple, se réjouir que le wagon quasi vide du train de ce matin lui ait permis de conserver sans problème la «distance sociale». Ou la nécessité de développer rapidement un vaccin contre ce nouveau coronavirus.

\section{Et un jour à nouveau pouvoir s'enlacer}

Il avait déjà constaté le développement insatisfaisant de substances et vaccins contre les maladies virales au moment de la crise Ebola en 2015. Alors doyen de la faculté de médecine, il avait initié avec les hôpitaux universitaires un centre des maladies virales émergentes avec une nouvelle chaire. Bien sûr, la menace que font peser sur le monde ces virus émergents n'est pas nouvelle: le Moyen Age a connu les ravages de la peste et après la Première Guerre mondiale a sévi la terrible "grippe espagnole». Il s'interrompt brièvement et ajoute d'un ton narquois: «La grippe espagnole nous est venue avec les soldats américains - peut-être Donald Trump ferait-il bien de s'en rappeler aujourd'hui.» A propos de la pandémie en cours, le professeur de médecine avance un pronostic prudent, mais optimiste: «Nous allons atteindre le pic dans les prochaines semaines - et puis, la vague fléchira. Je ne crois pas que je ne pourrai plus jamais enlacer mes petits-enfants.»

Crédits photos

Daniel Lüthi 